\title{
Scalar suppression on large scales in open inflation
}

AUTHOR(S):

White, Jonathan; Zhang, Ying-li; Sasaki, Misao

\section{CITATION:}

White, Jonathan ... [et al]. Scalar suppression on large scales in open inflation. Physical Review D 2014, 90(8): 083517.

ISSUE DATE:

2014-10-16

URL:

http://hdl.handle.net/2433/191206

RIGHT:

C 2014 American Physical Society 
PHYSICAL REVIEW D 90, 083517 (2014)

\title{
Scalar suppression on large scales in open inflation
}

\author{
Jonathan White, ${ }^{*}$ Ying-li Zhang, ${ }^{\dagger}$ and Misao Sasaki ${ }^{\ddagger}$ \\ Yukawa Institute for Theoretical Physics, Kyoto University, Kyoto 606-8502, Japan \\ (Received 15 August 2014; published 16 October 2014)
}

\begin{abstract}
We consider two toy models of open inflation and investigate their ability to give a suppression of scalar power on large scales while also satisfying observational constraints on the spatial curvature of the Universe. Qualitatively we find that both models are indeed capable of fulfilling these two requirements, but we also see that effects of the quantum tunneling must be carefully taken into account if we wish to make quantitative predictions.
\end{abstract}

DOI: 10.1103/PhysRevD.90.083517

PACS numbers: $98.80 . \mathrm{Cq}, 98.70 . \mathrm{Vc}$

\section{INTRODUCTION}

Precision measurements of the cosmic microwave background (CMB) by the WMAP and Planck collaborations are in very good agreement with what has become the standard model of cosmology, namely inflation plus $\Lambda \mathrm{CDM}$ [1-3]. There are, however, some anomalies, such as a $5-10 \%$ deficit in the temperature power spectrum on large scales $(l \lesssim 40)$ [4]. While the statistical significance of this anomaly is currently only $2.5-3 \sigma$, the tension will be exacerbated if the recent findings of the BICEP2 teamwho have reported a tensor-to-scalar ratio of $r \sim \mathcal{O}(0.1)$ [5] -are confirmed $[6,7]$. The reason for this increased tension is easy to understand: the temperature power spectrum on large scales $(l \lesssim 100)$ receives contributions from both scalar and tensor perturbations, meaning that for a given observed power the scalar contribution must be suppressed if there exists a non-negligible tensor contribution.

Perhaps the simplest way in which a suppression on large scales can be accommodated is to allow for a negative running of the spectral index, defined as $\alpha_{s} \equiv d n_{s} / d \ln k$. What one finds, however, is that the required value of $\alpha_{s}$ is $\sim \mathcal{O}(0.01)[5,8]$, which is 1 or 2 orders of magnitude larger than the running predicted in standard slow-roll models of inflation. Moreover, a large running also tends to spoil the fit to data on small scales. As such, it appears that a nonpower-law scalar spectrum may be preferred $[8,9]$, namely one that is able to give a localized suppression on large scales. Note that one can also try to alleviate tensions between the BICEP2 results and those of Planck by allowing for a modified tensor spectrum [7,10,11], but here we focus on modifying the scalar spectrum.

In canonical single-field models of inflation the power spectrum takes the simple form

$$
\mathcal{P}_{\mathcal{R}}(k)=\left.\frac{H^{4}}{4 \pi^{2} \dot{\phi}^{2}}\right|_{k=a H},
$$

\footnotetext{
jwhite@yukawa.kyoto-u.ac.jp yingli@yukawa.kyoto-u.ac.jp *misao@yukawa.kyoto-u.ac.jp
}

where the subscript $k=a H$ denotes the fact that the quantity should be evaluated at the time when the scale $k$ left the Hubble horizon. Given the inverse dependence on $\dot{\phi}$, a natural way in which we might seek to suppress the spectrum would be to require that the field be rolling quickly during the period that large scales left the horizon, i.e. during the first few observable $e$-foldings of inflation. ${ }^{1}$ This mechanism for suppression has been considered in the literature (see e.g. Refs. [12,16-19]), and in canonical single-field inflation models an enhancement of $\dot{\phi}$ is associated with a steepening of the potential towards the onset of inflation. While such a tuned steepening might seem unnatural, this is not necessarily the case in the context of open inflation [6,20-24].

Models of open inflation involve potentials of the form shown in Fig. 1, and the last stage of observable inflation is preceded by tunneling from a false vacuum described by a Coleman-De Luccia (CDL) instanton [25]. The universe nucleated in the tunneling process has negative curvature, i.e. it is open. In general, CDL instantons require $\left|V_{\phi \phi}\right|>$ $H^{2}$ during the tunneling process [26], which is in contradiction with the requirement $\left|V_{\phi \phi}\right| \ll H^{2}$ for slow-roll inflation. As such, it is expected that between the end of the tunneling process and the onset of standard slow-roll inflation one has an intervening stage of "fast-roll" inflation, during which the potential becomes progressively less steep. In order that observable signatures of this fast-roll phase remain, we require that the ensuing stage of slow-roll inflation does not last too long. If the number of $e$-foldings is too small, however, then the model becomes tightly constrained by observational constraints on $\Omega_{\mathrm{K}}$ today. The compatibility of these two competing effects was discussed by Freivogel et al. and Bousso et al. [6,20-22]. It was found that models providing sufficient suppression of the scalar spectrum while satisfying bounds on $\Omega_{\mathrm{K}}$ are possible if the steepening of the potential towards the barrier is gradual enough.

\footnotetext{
${ }^{1}$ Note that there are other possible mechanisms; see e.g. Refs. [12-15].
} 


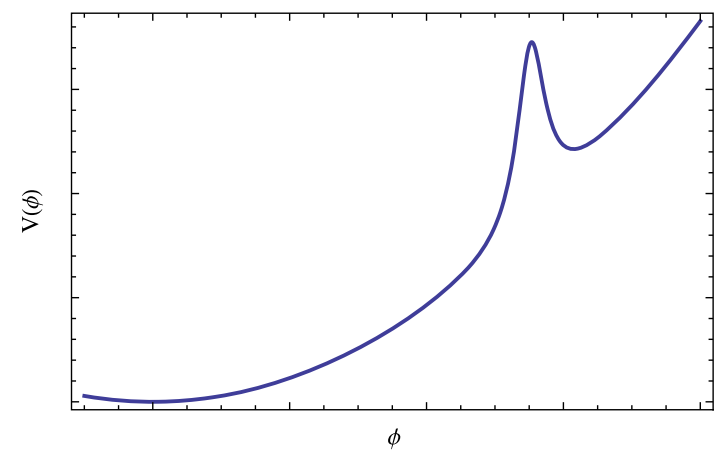

FIG. 1 (color online). The general form of the potential associated with open inflation models. Inflation is preceded by tunneling from the false vacuum and the steepening of the potential in the vicinity of the barrier leads to a suppression of the scalar power spectrum.

In this paper we revisit the toy models of open inflation presented in Refs. [23] and [24], and investigate their ability to produce a suppression of the large-scale scalar spectrum while evading observational constraints on $\Omega_{\mathrm{K}}$. In Sec. II we begin by giving a brief summary of open inflation and present the relevant background and perturbation equations. In Sec. III we then analyze two of the toy models discussed in Ref. [23]. Based on the two toy models, in Sec. IV we then summarize more generally the nature of suppression from open inflation models and how they can be constrained using observations. Finally we conclude in Sec. V.

\section{OPEN INFLATION}

To describe the tunneling process preceding single-field open inflation, we consider a class of model whose effective potential $V(\phi)$ has a local minimal at $\phi_{0}$ and global minimum at $\phi=0$ with $V(0)=0$, as depicted in Fig. 1. The action is given by

$$
\mathcal{S}=\int \sqrt{-g} d^{4} x\left[\frac{R}{2}-\frac{1}{2} g^{\mu \nu} \partial_{\mu} \phi \partial_{\nu} \phi-V(\phi)\right] .
$$

Note that here and throughout the paper we set $8 \pi G=1 /$ $M_{\mathrm{Pl}}^{2}=1$.

Conventionally, the instanton solution is explored under the assumption of $\mathcal{O}(4)$ symmetry, since it has been proved that the $\mathcal{O}(4)$-symmetric solution gives the lowest value of the Euclidean action for a wide class of scalar-field theories [27]. Hence, this solution is exponentially favored when calculating the corresponding tunneling probability. It is also reasonable to use this assumption with gravity included [25]. To be specific, the metric of an $\mathcal{O}(4)$ invariant Euclidean spacetime can be written as

$$
d s_{\mathrm{E}}^{2}=d \xi^{2}+a_{\mathrm{E}}^{2}(\xi)\left(d \chi_{\mathrm{E}}^{2}+\sin ^{2} \chi_{\mathrm{E}} d \Omega_{2}^{2}\right) .
$$

The corresponding Euclidean background equations are given as

$$
\begin{gathered}
H_{\mathrm{E}}^{2}=\frac{1}{3}\left(\frac{\dot{\phi}^{2}}{2}-V\right)+\frac{1}{a_{\mathrm{E}}^{2}}, \\
\ddot{\phi}+3 H_{\mathrm{E}} \dot{\phi}-V_{\phi}=0,
\end{gathered}
$$

where a dot denotes differentiation with respect to $\xi$ and $H_{\mathrm{E}} \equiv \dot{a}_{\mathrm{E}} / a_{\mathrm{E}}$. For the CDL instanton solution, we choose $\xi=0$ to coincide with the center of the nucleated $\mathcal{O}(4)$ symmetric bubble, at which $\phi=\phi_{\text {exit }}, a_{\mathrm{E}}=0$ and $\dot{a}_{\mathrm{E}}=1$. We further set $\xi=\xi_{\mathrm{F}}(>0)$ in the false vacuum, where $\phi=\phi_{\mathrm{F}}, a_{\mathrm{E}}=0$ and $\dot{a}_{\mathrm{E}}=-1$.

On making an analytic continuation to the Lorentzian space corresponding to our open universe, the metric takes the form $[28,28-30]$

$$
\begin{aligned}
d s^{2} & =-d t^{2}+a^{2}(t)\left(d \chi^{2}+\sinh ^{2} \chi d \Omega^{2}\right) \\
& =a^{2}(\eta)\left(-d \eta^{2}+d \chi^{2}+\sinh ^{2} \chi d \Omega^{2}\right),
\end{aligned}
$$

where $t=-i \xi, a=-i a_{\mathrm{E}}, \chi=i \chi_{\mathrm{E}}$ and $a d \eta=d t$, and the background equations of motion become

$$
\begin{gathered}
H^{2}=\frac{1}{3}\left(\frac{1}{2} \dot{\phi}^{2}+V\right)+\frac{1}{a^{2}}, \\
\ddot{\phi}+3 H \dot{\phi}+V_{\phi}=0,
\end{gathered}
$$

where a dot now denotes taking the derivative with respect to $t$. The initial conditions coming from the instanton solution are $\phi=\phi_{\text {exit }}, \dot{\phi}=0, a=0$ and $\dot{a}=1$. Using these equations of motion and initial conditions, we then need to solve for the ensuing inflationary dynamics.

Locating the observer at the center of the spherical coordinates, the only tensor modes that contribute to the CMB temperature power spectrum are the even-parity modes, which can be expressed as

$$
\begin{aligned}
\delta g_{i j} & =a^{2} t_{i j} ; \\
t_{i j} & =\sum \hat{b}_{p l m} U_{p}(\eta) Y_{i j}^{(+) p l m}+\text { H.c. }
\end{aligned}
$$

Here $\hat{b}_{p l m}$ are the annihilation operators, $Y_{i j}^{(+) p l m}$ are the even-parity tensor harmonics on a unit 3-hyperboloid and $\eta$ is the conformal time. The $U_{p}(\eta)$ are found to satisfy [31]

$$
\mathcal{U}_{p}^{\prime \prime}+2 H U_{p}^{\prime}+\left(p^{2}+1\right) U_{p}=0,
$$

where a prime denotes $d / d \eta$ and $\mathcal{H}=a^{\prime} / a$.

The important scalar quantity is the comoving curvature perturbation $\mathcal{R}_{c}^{p}$, but this is more conveniently expressed in terms of a new variable $\boldsymbol{q}^{p}$ as

$$
\mathcal{R}_{c}^{p}=-\frac{\dot{\phi}}{2} \boldsymbol{q}^{p}-\frac{H}{a \dot{\phi}^{2}} \frac{d}{d t}\left(a \dot{\phi} \boldsymbol{q}^{p}\right) .
$$


The variable $\boldsymbol{q}^{p}$ then satisfies the simple equation

$$
\boldsymbol{q}^{p \prime \prime}-\left[\frac{\phi^{\prime 2}}{2}+\phi^{\prime}\left(\frac{1}{\phi^{\prime}}\right)^{\prime \prime}-\left(p^{2}+4\right)\right] \boldsymbol{q}^{p}=0 .
$$

The scalar and tensor power spectra are given in terms of $\mathcal{R}_{c}^{p}$ and $U_{p}$ as

$$
\mathcal{P}_{\mathcal{R}}=\frac{p^{3}}{2 \pi^{2}}\left|\mathcal{R}_{c}^{p}\right|^{2} \quad \text { and } \quad \mathcal{P}_{T}=\frac{p^{3}}{2 \pi^{2}}\left|U_{p}\right|^{2}
$$

respectively. In this work we fit the power spectra using the following functional forms:

$\mathcal{P}_{\mathcal{R}}=\left(\frac{H^{2}}{2 \pi \dot{\phi}}\right)_{t=t_{\mathcal{R}, p}}^{2} \frac{\cosh (\pi p)+\cos \left(\delta_{p}\right)}{\sinh (\pi p)} \frac{p^{2}}{c_{1}^{2}+p^{2}}$

$\mathcal{P}_{T}=4\left(\frac{H}{2 \pi}\right)_{t=t_{T, p}}^{2} \frac{\cosh (\pi p)-1}{\sinh (\pi p)} \frac{p^{2}}{c_{2}^{2}+p^{2}}$,

where the subscripts $t=t_{\mathcal{R}, p}$ and $t=t_{T, p}$ indicate that the quantity should be evaluated at the horizon-crossing time for scalar and tensor modes, respectively. The phase $\delta_{p}$ is irrelevant for large $p$ and behaves as $\delta_{p}-\pi \propto p$ for $p \rightarrow 0$. In fact, we take $\delta_{p}=\pi$ throughout, which corresponds to the case where maximal suppression is achieved. The above expressions for $\mathcal{P}_{\mathcal{R}}$ and $\mathcal{P}_{T}$ are based on analytic approximations derived in Refs. [31,32]. We can see that they differ from the standard expressions by the $p$-dependent "suppression factors," which tend to unity for large $p$ and $\pi p^{3} /\left(2 c_{1,2}^{2}\right)$ for $p \rightarrow 0$. This $p$-dependent suppression is distinct from that associated with the fast-roll dynamics, and reflects the systems memory of the quantum tunneling that preceded inflation [24]. The parameters $c_{1}$ and $c_{2}$ determine the scales at which this additional suppression becomes active for the scalar and tensor modes, respectively. Under the weak backreaction approximation, the spectra were found to take the above form with $c_{1}=1$ and $c_{2}=1$ [31,32]. In Appendix $\mathrm{C}$ of Ref. [31], similar expressions were also found for an analytically soluble model, but with $c_{1}=2$ and $c_{2}=1$. In this paper we take the two parameters $c_{1}$ and $c_{2}$ as free parameters that we use to fit the numerical results of Ref. [24].

In order to determine the horizon-crossing conditions we return to the equations of motion. Firstly, for $U_{p}$, we see from Eq. (10) that horizon crossing takes place when $a^{2} H^{2}=p^{2}+1$, as in the standard case. In order to determine $t_{\mathcal{R}, p}$, we first need to use Eqs. (11) and (12) to find an equation of motion for $\mathcal{R}_{c}^{p}$. We find ${ }^{2}$

$$
\mathcal{R}_{c}^{p \prime \prime}+2 A(\eta, p) \mathcal{R}_{c}^{p \prime}+B(\eta, p) \mathcal{R}_{c}^{p}=0,
$$

\footnotetext{
${ }^{2}$ This equation can also be derived using the action for $\mathcal{R}_{c}$ given in Appendix B of Ref. [33].
}

with

$$
\begin{aligned}
& A(\eta, p)=\frac{k^{2} \mathcal{H}^{2} \frac{\mathcal{H}}{a \phi^{\prime}}\left(\frac{a \phi^{\prime}}{\mathcal{H}}\right)^{\prime}-\frac{\phi^{\prime 2}}{2} \mathcal{H}}{k^{2} \mathcal{H}^{2}-\frac{\phi^{\prime 2}}{2}}, \\
& B(\eta, p)=\frac{k^{4} \mathcal{H}^{2}+\left(1+\frac{\mathcal{H}^{2}}{\phi^{2}}\left(\frac{\phi^{\prime 2}}{\mathcal{H}}\right)^{\prime}\right) k^{2}-\frac{\phi^{\prime 2}}{2}}{k^{2} \mathcal{H}^{2}-\frac{\phi^{\prime 2}}{2}},
\end{aligned}
$$

where $k^{2}=p^{2}+4$. Note that in the limit of large $p(\leftrightarrow$ large $k$ ) the equation of motion for $\mathcal{R}_{c}^{p}$ reduces to the standard form, where, defining $z=a \phi^{\prime} / \mathcal{H}, A(\eta, p)=z^{\prime} / z$ and $B(\eta, p)=p^{2}$. Defining horizon crossing as when $A(\eta, p)^{2}=B(\eta, p)$, noting $k^{2} \geq 4$ and assuming $\dot{\phi}^{2} / H^{2} \ll$ 1 and $1 /\left(a^{2} H^{2}\right) \ll 1$ (which is always the case after curvature domination in our particular models), we find the condition

$$
p^{2}+4=a^{2} H^{2}\left(1+\frac{\ddot{\phi}}{\dot{\phi} H}\right)^{2}-\left(1+2 \frac{\ddot{\phi}}{\dot{\phi} H}\right) .
$$

One can see that if the slow-roll condition $\ddot{\phi} /(\dot{\phi} H) \ll 1$ is satisfied, then this reduces to the standard horizon-crossing condition for large $p$.

\section{TOY MODELS}

Having summarized the key features of open inflation models and the relevant background and perturbation equations, in this section we revisit two of the toy models that were introduced and analyzed numerically in Ref. [24].

\section{A. Model 1}

The potential of Model 1 takes the form

$$
V(\phi)=\frac{1}{2} m^{2} \phi^{2}\left(1+\frac{\alpha^{2}}{\beta^{2}+(\phi-\nu)^{2}}\right) .
$$

We consider the same parameter values as considered in Ref. [24], except we work in reduced Planckian units instead of Planckian units. As such, our parameters are given as

$$
\begin{aligned}
\nu & =3.5 \times \sqrt{8 \pi}, \\
\beta^{2} & =2 \alpha^{2}, \\
\beta & =0.1 \times \sqrt{8 \pi}, \\
m & =1.5 \times 10^{-6} \times \sqrt{8 \pi} .
\end{aligned}
$$

By solving Eqs. (4) and (5) numerically, one is able to find a CDL instanton solution, which is plotted in the upper panel of Fig. 2. The value of the field at the end of the tunneling is given as 
JONATHAN WHITE, YING-LI ZHANG, AND MISAO SASAKI
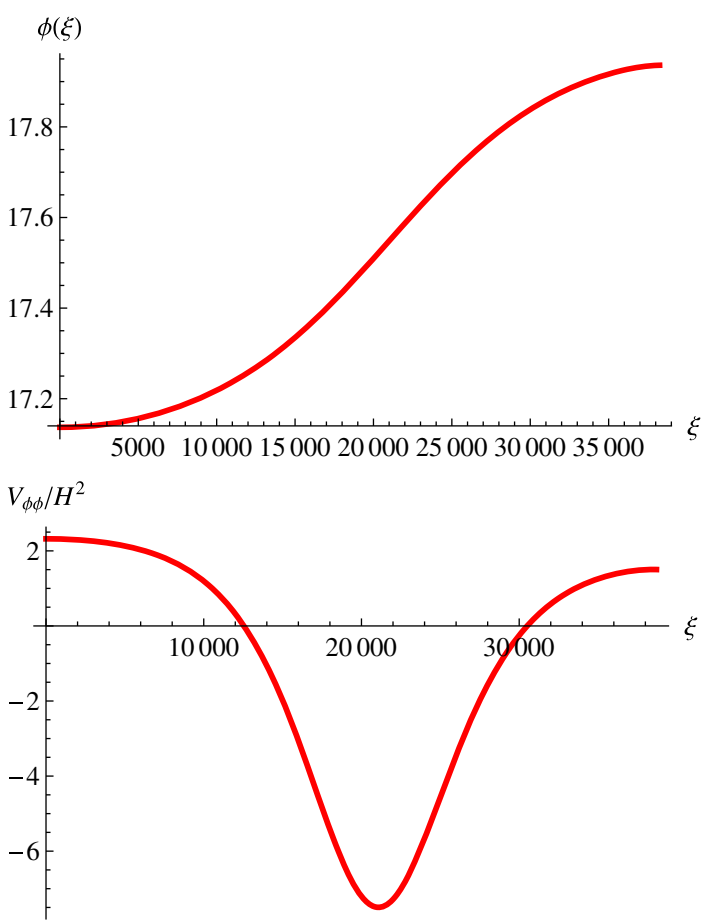

FIG. 2 (color online). Upper panel: $\phi$ as a function of $\xi$ for the CDL instanton solution of Model 1. Lower panel: A plot of $\left|V_{\phi \phi}\right| / H^{2}$ as a function of $\xi$ for the CDL instanton solution of Model 1. We can see that for most of the trajectory the condition $\left|V_{\phi \phi}\right|>H^{2}$ is satisfied.

$$
\phi_{\text {exit }}=17.14 \text {. }
$$

In the lower panel of Fig. 2 we plot $\left|V_{\phi \phi}\right| / H^{2}$ along the CDL instanton trajectory, and we see that $\left|V_{\phi \phi}\right|>H^{2}$ is indeed satisfied for most of the trajectory.

Using the value of $\phi_{\text {exit }}$ found above, we then solve the background equations of motion (7) and (8) for $\phi$ and $a$ after the tunneling. In Fig. 3 we plot the resulting evolution of the Hubble parameter as a function of the number of $e$-foldings before the end of inflation (solid black curve). ${ }^{3}$ We also plot three scales of interest, which are

(1) the curvature scale, corresponding to $p=1$ (blue dashed curve),

(2) the upper limit on the current Hubble scale, as determined by the constraint $\Omega_{\mathrm{K}} \lesssim 0.01$, i.e. $p_{H_{0}}=$ $1 / \sqrt{\Omega_{\mathrm{K}}} \simeq 10$ (red dot-dashed curve), and

(3) the scale that approximately corresponds to the CMB multipole $l=100$, as determined assuming that $H_{0}$ is given by the upper limit mentioned above (green dotted curve).

Finally, the times at which the last two of the aforementioned scales leave the horizon (as simply determined by $p=a H)$ are marked with vertical dashed lines approximately 64 and $60 e$-foldings before the end of inflation,

\footnotetext{
${ }^{3}$ The end of inflation is taken to be when $\epsilon_{V} \equiv(1 / 2)$ $\left(V_{\phi} / V\right)^{2}=1$.
}

PHYSICAL REVIEW D 90, 083517 (2014)

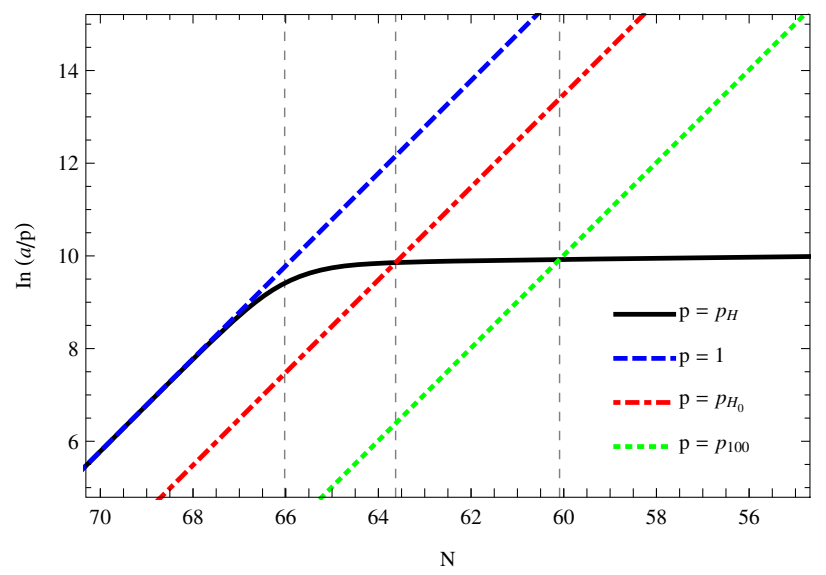

FIG. 3 (color online). Evolution of the Hubble scale as a function of the number of $e$-foldings before the end of inflation (solid black curve). Also plotted are the evolution of the curvature scale (upper dashed line), the current Hubble scale as determined assuming that the observational constraint on $\Omega_{\mathrm{K}}$ is saturated (middle dot-dashed line) and the scale associated with $l=100$ (bottom dotted line). The left-most vertical dashed line corresponds to the time at which the curvature term becomes subdominant to the potential term in the Friedmann equation. The next two lines, from left to right, indicate the times at which the current Hubble scale and the scale associated with $l=100$ left the Hubble horizon.

respectively. The third, left-most vertical dashed line, located approximately $66 e$-foldings before the end of inflation, corresponds to the time of potential-curvature equality. Unless otherwise mentioned, the vertical dashed lines shown in all following plots will correspond to these same three events. As we will see later, it is also important to know the time at which the curvature becomes subdominant to the kinetic component of $H^{2}$. Interestingly, in Model 1 this time almost exactly coincides with the time at which the scale $p_{H_{0}}$ left the horizon, i.e. $\sim 64 e$-foldings before the end of inflation. The evolution of the three components of $H^{2}$ are plotted explicitly in Fig. 4.

We emphasise that the $p_{H_{0}}$ discussed above corresponds to the current Hubble scale as determined assuming that the observational constraint on $\Omega_{\mathrm{K}}$ is saturated. For our choice of model parameters, we see that this scale would leave the horizon approximately $64 e$-foldings before the end of inflation. However, this would seem a little too early, as standard arguments dictate that the current horizon scale left the horizon $\sim 60 e$-foldings before the end of inflation [34].

In order to help relate features in the background dynamics and power spectrum to the underlying potential, in Fig. 5 we indicate the location of the scalar field on the potential at the three times discussed above. Note that the order of the vertical dashed lines is the reverse of that in other plots. Looking at the form of the potential (20), we see that it naturally lends itself to being decomposed into a fiducial $m^{2} \phi^{2}$ potential and a correction to this around the 
SCALAR SUPPRESSION ON LARGE SCALES IN OPEN ...

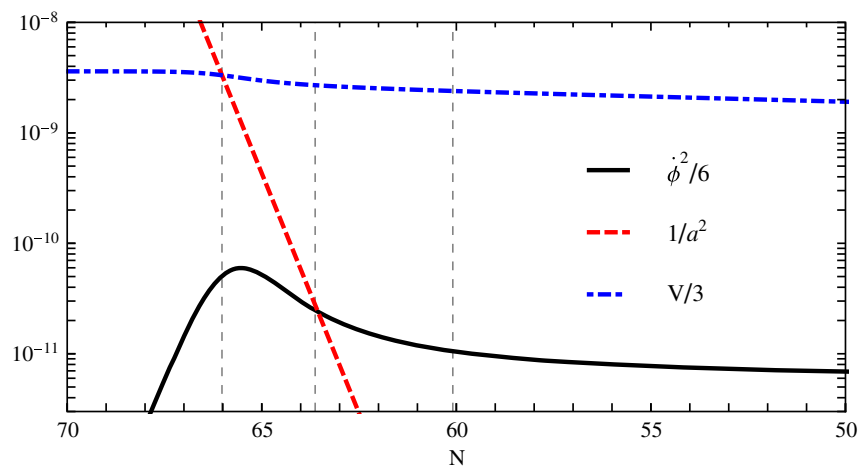

FIG. 4 (color online). Evolution of the kinetic (solid black curve), curvature (dashed red curve) and potential (dot-dashed blue curve) contributions to $H^{2}$ as a function of the number of $e$-foldings before the end of inflation. The three vertical dashed lines are as in Fig. 3.

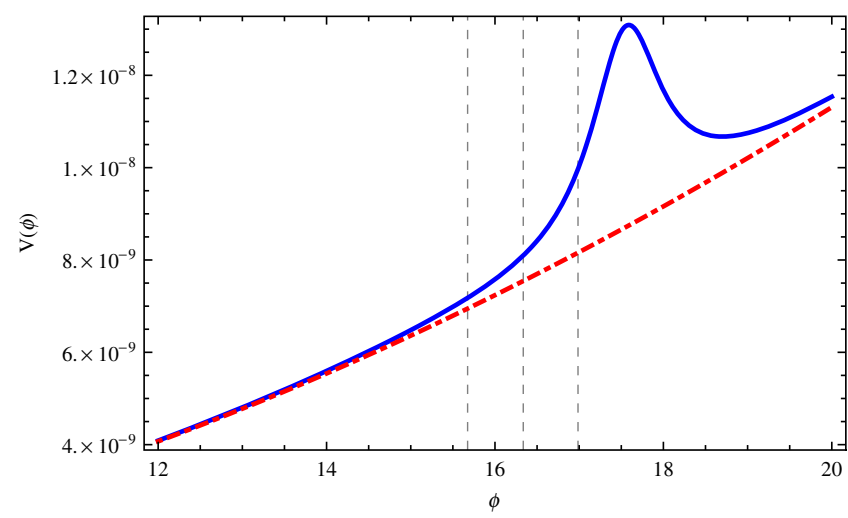

FIG. 5 (color online). Plot of the potential in Model 1 of Ref. [24], with the parameter choice shown in the main text. The three vertical lines are as in Fig. 3, except their order from left to right is reversed.

tunneling barrier. As such, in Fig. 5 we also plot the fiducial $m^{2} \phi^{2}$ potential. We see that the steepening of the potential relative to $m^{2} \phi^{2}$ remains non-negligible until after the scale $p_{100}$ leaves the horizon. As such, we expect that observable scales will indeed be subject to suppression in this model.

In the upper panel of Fig. 6 we compare the evolution of the three slow-roll parameters $\epsilon \equiv-\dot{H} / H^{2}, \epsilon_{V} \equiv$ $\left(V_{\phi} / V\right)^{2} / 2$ and $\epsilon_{m}=2 / \phi^{2}$ with the evolution of $\dot{\phi}^{2} / 2 H^{2}$. As we can see from Eq. (14), the quantity that we are interested in evaluating is $\mathcal{P}_{\mathcal{R}} \propto H^{4} / \dot{\phi}^{2}$. In the case of a flat universe, this can be reexpressed in terms of $\epsilon$ as $\mathcal{P}_{\mathcal{R}} \propto H^{2} / \epsilon$, which in turn, under the slow-roll approximation can be reexpressed as $\mathcal{P}_{\mathcal{R}} \propto H^{2} / \epsilon_{V}$. In the case of open inflation, however, we have $\dot{H}=-\dot{\phi}^{2} / 2-1 / a^{2}$, meaning that we can no longer directly replace $\dot{\phi}^{2}$ with $\epsilon$. Moreover, as the slow-roll condition may be violated in the early stages of evolution after tunneling, we are also not necessarily able to make the second approximation $\epsilon \simeq \epsilon_{V}$. Of course, at late times we expect the curvature to become
PHYSICAL REVIEW D 90, 083517 (2014)

negligible and for slow-roll inflation to take place, so it is interesting to explicitly see at what stage the equivalence of the three quantities is recovered. As we can see from Fig. 6, the equivalence is recovered approximately $63 e$-foldings before the end of inflation. This time is shortly after that at which the curvature term becomes negligible in comparison to both the potential and kinetic terms in the Friedmann equation. This is exactly as we would expect in light of the contribution of $1 / a^{2}$ to $\dot{H}$. The fact that $\epsilon$ and $\epsilon_{V}$ also become approximately equal at this time tells us that the potential must already be relatively flat and the slow-roll approximation is a good one. Another point to note from this figure is the difference between $\epsilon$ and $\dot{\phi}^{2}$ at early times. As a result of the initial conditions imposed by the CDL instanton and the initial curvature domination, $\epsilon$ asymptotes to unity while $\dot{\phi}$ vanishes. This is evidently very important in determining the asymptotic behavior of $\mathcal{P}_{\mathcal{R}}$, as we will see shortly. Finally the $\epsilon_{m}$ that we have plotted corresponds to the slow-roll parameter associated with the fiducial $m^{2} \phi^{2}$ potential. We can see that even $60 e$-foldings before the end of inflation there is a non-negligible difference between $\epsilon_{m}$ and $\epsilon_{V}$.

To leading order in the slow-roll approximation, the tilt of the power spectrum is given as

$$
n_{s}-1=-2 \epsilon-\eta,
$$

where $\eta \equiv \dot{\epsilon} /(H \epsilon)$. Using the fact that $\eta_{V} \equiv V_{\phi \phi} / V \simeq$ $-\eta / 2+2 \epsilon$, this can be written in the perhaps more familiar form

$$
n_{s}-1=-6 \epsilon_{V}+2 \eta_{V}
$$

We know that the initial conditions prescribed by the CDL instanton dictate that $\eta_{V}$ be large, which will tend to give a blue-tilted spectrum. Given that the spectrum must be red-tilted on small scales, we are therefore interested in following the evolution of $\eta$ and in determining when the transition from a blue- to a red-tilted spectrum takes place. As such, in the lower panel of Fig. 6 we plot the evolution of $\tilde{\eta} \equiv-\eta / 2+2 \epsilon, \eta_{V}$ and $\eta_{m} \equiv 2 / \phi^{2}$, where the last quantity corresponds to the equivalent slow-roll parameter associated with the fiducial $m^{2} \phi^{2}$ potential. Similar to the case with $\epsilon$ and $\epsilon_{V}$, we note the different behavior of $\tilde{\eta}$ and $\eta_{V}$ at early times. This is again due to the initial conditions imposed by the CDL instanton and the domination of curvature, which give $\eta=0$ and $\epsilon=1$ as $t \rightarrow 0$. Comparing with the upper panel of Fig. 6, we also note that the time at which the three different definitions of $\eta$ become equivalent is somewhat later than the corresponding time for $\epsilon$.

In Fig. 7 we plot the scalar and tensor power spectra associated with Model 1 as functions of the comoving scale $p$. In the case of the scalar spectrum we plot four different curves. Three of these correspond to different variants of the analytic expression (14), namely (1) the full 

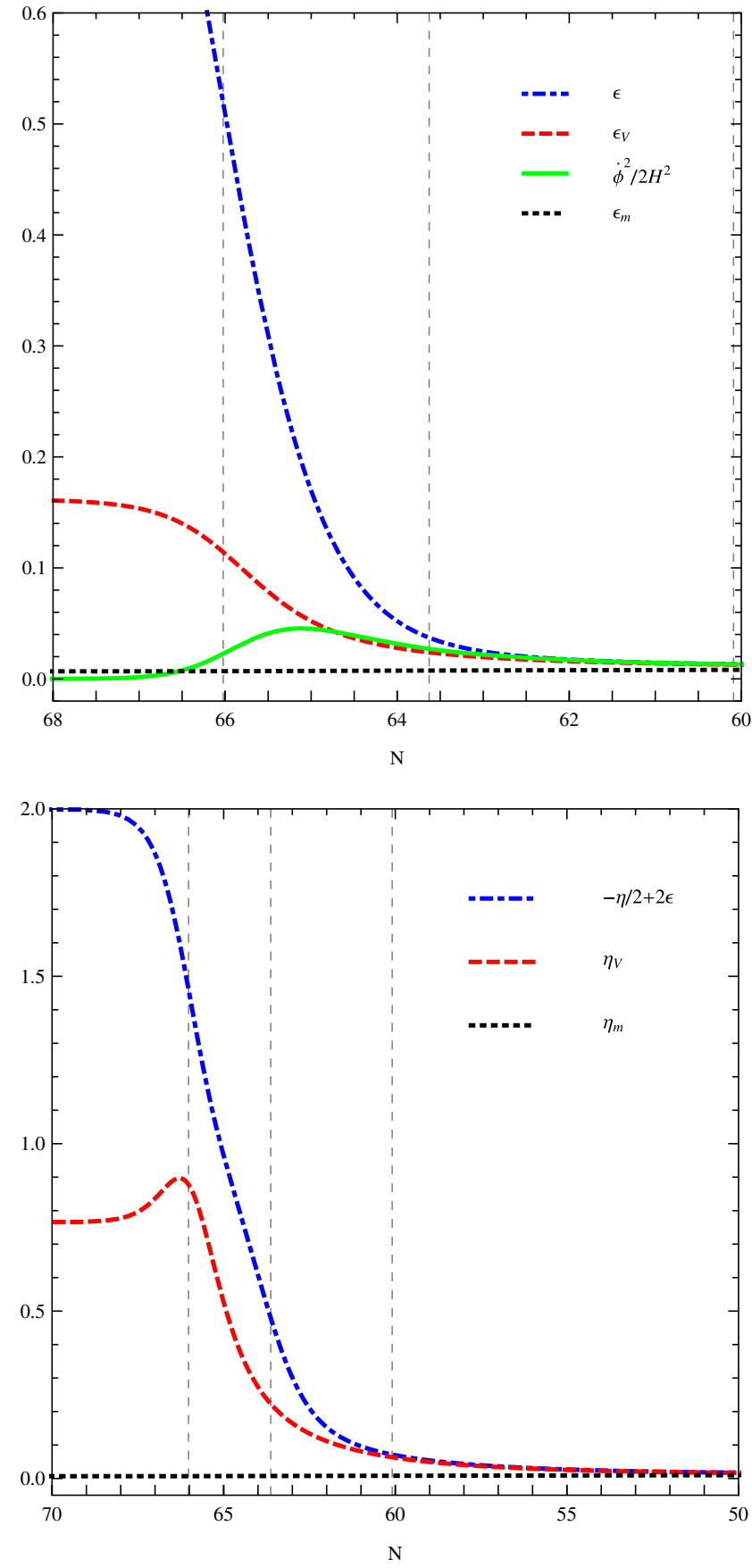

FIG. 6 (color online). Upper panel: Evolution of the four slowroll quantities $\epsilon$ (blue dot-dashed curve), $\epsilon_{V}$ (red dashed curve), $\epsilon_{m}$ (black dotted curve) and $\dot{\phi}^{2} / 2 H^{2}$ (solid green curve) as a function of the number of $e$-foldings before the end of inflation. Lower panel: Evolution of the three slow-roll quantities $\tilde{\eta} \equiv-\eta / 2+2 \epsilon$ (blue dot-dashed curve), $\eta_{V}$ (red dashed curve) and $\eta_{m}$ (black dotted curve) as a function of the number of $e$-foldings before the end of inflation. In both panels the three vertical lines are as in Fig. 3.

expression (14) (blue solid curve), (2) Eq. (14) without the $p$-dependent suppression factor that distinguishes Eq. (14) from the standard flat-universe expression (red dot-dashed curve) and (3) Eq. (14) taking $t_{\mathcal{R}, p}$ as the time when

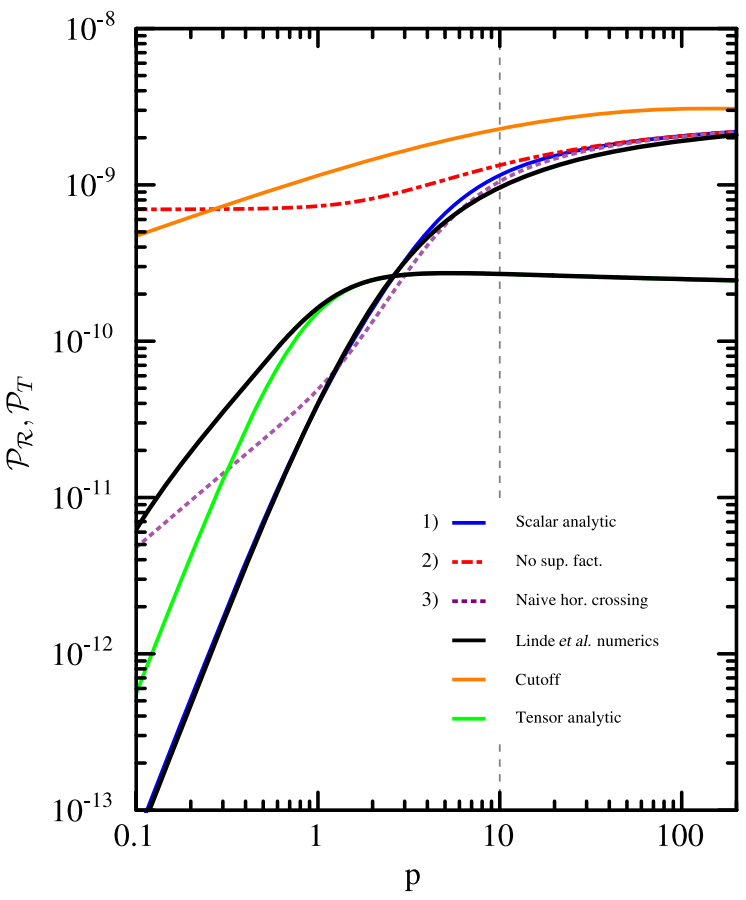

FIG. 7 (color online). The scalar and tensor power spectra as functions of the comoving scale $p$. In the case of the scalar spectrum we plot three variants of the analytic expression (14), namely (1) the full expression (solid blue curve), (2) Eq. (14) without the $p$-dependent suppression factor that distinguishes Eq. (14) from the standard flat-universe expression (red dotdashed curve) and (3) Eq. (14) taking $t_{\mathcal{R}, p}$ as the time when $a^{2} H^{2}=p^{2}+1$ instead of when Eq. (19) is satisfied (purple dotted curve). We also plot the numerical solution for $\mathcal{R}_{c}^{p}$ from Ref. [24] (upper solid black curve). The fitted curves use $c_{1}=4$. Finally, for comparison, we also plot the form of the spectrum associated with the cutoff model considered in Ref. [3] (solid orange curve). In the case of the tensor spectrum we simply plot the full expression (15) with $c_{2}=1$ (solid green curve) and the numerical solution from Ref. [24] (lower solid black curve). The vertical dashed line at $p=10$ corresponds to the current Hubble scale if we assume that observational constraints on $\Omega_{\mathrm{K}}$ are saturated.

$a^{2} H^{2}=p^{2}+1$ instead of when the condition (19) is satisfied (purple dotted curve). The upper solid black curve then corresponds to the numerical solution for $\mathcal{R}_{c}^{p}$ found in Ref. [24]. We take $c_{1}=4$ in Eq. (14), which we find to give the best fit to the numerical results for small $p$. Qualitatively, we see that the full analytic result (solid blue curve) matches the numerics well, especially for small and large $p$. At intermediate scales, around $p \simeq 10$, however, it tends to underestimate the amount of suppression, with the discrepancy being on the order of $10 \%$. If we assume that the observational bound on $\Omega_{\mathrm{K}}$ is saturated, this scale coincides exactly with the current Horizon scale. As such, if we are interested in quantitatively constraining the suppression from open inflation, we see that numerical calculations are necessary. 


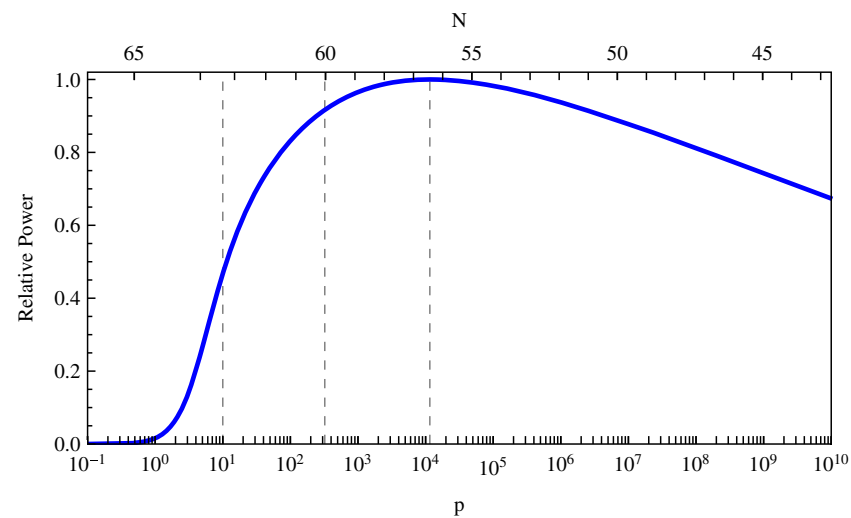

FIG. 8 (color online). The magnitude of the scalar power relative to the magnitude at the scale of transition between a blue- and red-tilted spectrum. In this plot the vertical dashed lines, from left to right, correspond, respectively, to (1) the current Horizon scale $p_{H_{0}}$ as determined assuming that $\Omega_{\mathrm{K}}=0.01$, (2) the scale associated with $l=100, p_{100}$, and (3) the scale at which the spectrum transitions from being blue- to red-tilted, $p_{\text {red }}$. On the upper horizontal axis we also give the number of $e$-foldings before the end of inflation that each given scale $p$ leaves the horizon.

From curves (2) and (3) we are able to appreciate the importance of corrections to the standard flat-universe expression for $\mathcal{P}_{\mathcal{R}}$ for small $p$. The fact that curve (2) always underestimates the suppression of the scalar spectrum highlights the fact that the fast rolling of the inflaton is not the only source of suppression, especially on very large scales. The additional suppression reflects the system's memory of the tunneling process preceding inflation [24]. We also see that curve (3) underestimates the suppression for $p \lesssim 1$. This reflects the fact that largescale modes freeze out at later times than predicted by the standard horizon-crossing condition, leading to a suppression in their amplitude. As expected, all curves converge for large $p$, where the effects of the tunneling become negligible.

In addition to the four curves discussed above, for comparison we also plot the spectrum associated with the so-called cutoff model that has been considered in the literature; see e.g. Refs. [12,13]. The model-corresponding to the solid orange curve in Fig. 7-is a phenomenological example of a spectrum with suppression on large scales, and takes the form

$$
\mathcal{P}_{\text {co }}=A_{s}\left(\frac{p}{p_{*}}\right)^{n_{s}-1}\left[1-\exp \left\{-\left(\frac{p}{p_{c}}\right)^{\lambda_{c}}\right\}\right] .
$$

The Planck team found that such a form for the spectrum was preferred over power-law $\Lambda \mathrm{CDM}$ with $2 \Delta \ln \mathcal{L}_{\max }=$ 2.9 [3]. The corresponding best-fit parameters were $\ln \left(p_{c} /\left(a_{0} \mathrm{Mpc}^{-1}\right)\right)=-8.493$ and $\lambda_{c}=0.474$, where the pivot scale $p_{*} / a_{0}=0.05 \mathrm{Mpc}^{-1}$ was used. While it is not our intention that our models quantitatively fit the data well, it is nevertheless interesting to qualitatively compare our spectra with this cutoff model. For the orange curve in Fig. 7 we have used Planck's best-fit values for $p_{c} / a_{0}$ and $\lambda_{c}$ and further assumed that $\Omega_{\mathrm{K}}=0.01$ and $n_{s}=0.96$. The amplitude $A_{s}$ is adjusted so that our spectrum and the phenomenological model agree at $p=10^{5}$, as both spectra have relaxed to the standard power-law form on these scales.

In comparing Model 1 with the cutoff spectrum, the main feature we note is that Model 1 gives substantially more suppression. This feature is favorable in light of the fact that Planck's best-fit spectrum was found assuming no tensor modes. In order to be able to accommodate a tensor contribution to large-scale CMB temperature fluctuations (as suggested by BICEP2), we expect that a larger suppression of the scalar spectrum will be required. The discrepancy between the two models is, however, rather substantial, even at $p \simeq 100$. Better agreement could perhaps be achieved by correcting our assumption that $\Omega_{\mathrm{K}}=0.01$. As such, in Model 1 we may find that the bestfit value for $\Omega_{\mathrm{K}}$ is unobservably small.

For reference, we also plot the tensor spectrum, but this time only include the full analytic result given by Eq. (15) with $c_{2}=1$ (solid green curve) and the numerical results from Ref. [24] (lower solid black curve). It is clear to see that the onset of suppression occurs at much smaller $p$ in the case of the tensor spectrum, i.e. on scales larger than the current Hubble horizon. In contrast with the scalar spectrum, the tensor spectrum is not affected by the fast rolling of the inflaton. As such, the suppression on large scales is purely a result of the preinflationary tunneling and the presence of the bubble wall. See Ref. [24] for a more detailed discussion.

Finally, in Fig. 8 we plot the magnitude of the scalar power relative to that at the scale at which the spectrum transitions from being blue- to red-tilted. This transition occurs approximately $56 e$-foldings before the end of inflation, when the scale $p_{\text {red }} \simeq 10^{4}$ left the horizon. Given that potential-curvature equality occurs approximately 66 $e$-foldings before the end of inflation, we find that we have roughly $10 e$-foldings of the "fast-roll" phase before the spectrum becomes red-tilted and standard slow-roll is achieved.

Even in the best-case scenario, it is only possible to detect a $\Omega_{\mathrm{K}} \gtrsim 10^{-4}$, which would correspond to $p_{H_{0}}=10^{2}$. As such, we see that in Model 1 we have the possibility that suppression of the scalar spectrum is observed on large scales while $\Omega_{\mathrm{K}}$ remains undetectable. Indeed, if we were to make the usual assumption that $p_{H_{0}}$ leaves the horizon $\sim 60$ $e$-foldings before the end of inflation, this would correspond to $p_{H_{0}} \sim 3 \times 10^{2}$, giving an unobservable $\Omega_{\mathrm{K}} \sim 1 \times 10^{-5}$. However, in such a scenario the scale $p_{\text {red }}$ would coincide with $l \sim 100$, and suppression on the order of $10 \%$ would be observed at scales corresponding to the current Hubble horizon. 


\section{B. Model 2}

The second model considered in Ref. [24] has a potential of the form

$$
V(\phi)=\frac{m^{2}}{2}\left(\phi^{2}-B^{2} \frac{\sinh [A(\phi-\nu)]}{\cosh ^{2}[A(\phi-\nu)]}\right)
$$

with parameters chosen as

$$
\begin{aligned}
m & =10^{-6} \times \sqrt{8 \pi}, \\
A & =20 / \sqrt{8 \pi}, \\
B & =4 \times \sqrt{8 \pi}, \\
\nu & =3.5 \times \sqrt{8 \pi} .
\end{aligned}
$$

The CDL instanton solution and the evolution of $V_{\phi \phi} / H^{2}$ through the tunneling process are shown in Fig. 9. From the upper plot we are able to obtain $\phi_{\text {exit }}=16.55$, and from the lower plot we see that the
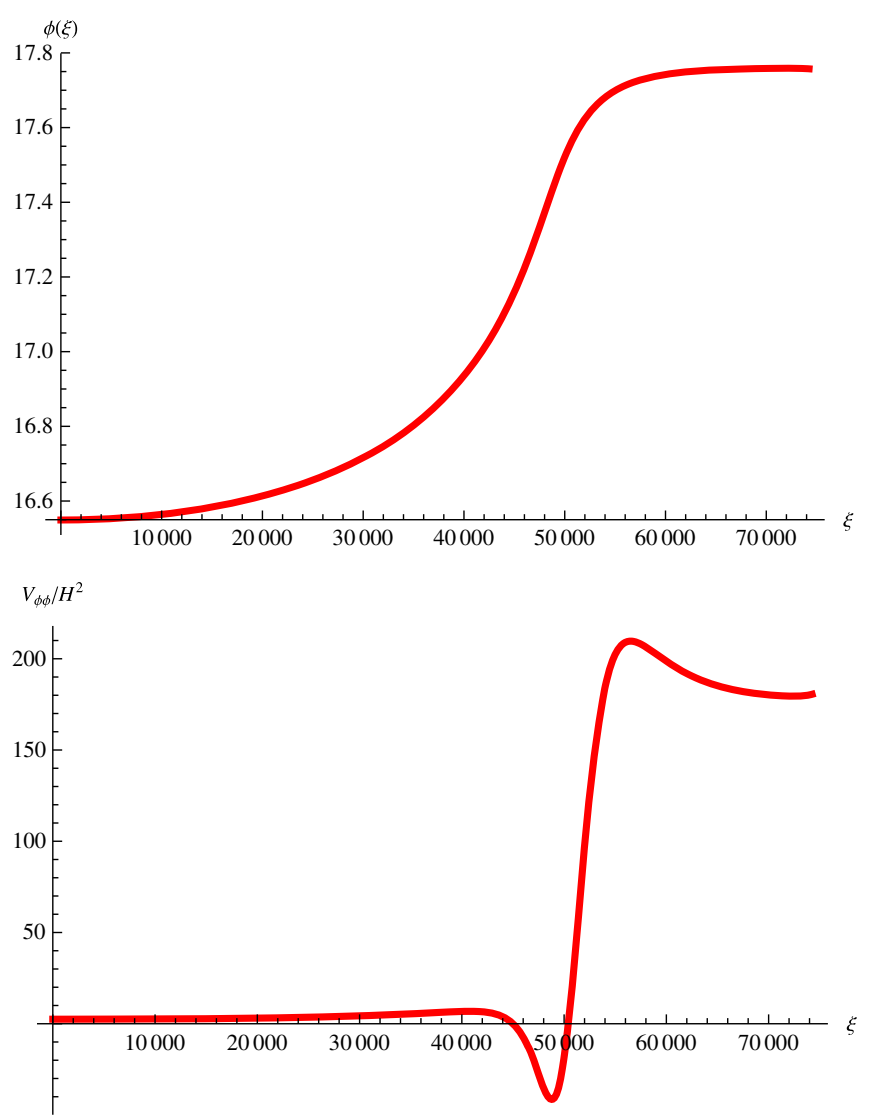

FIG. 9 (color online). Upper panel: $\phi$ as a function of $\xi$ for the CDL instanton solution of Model 2. Lower panel: A plot of $\left|V_{\phi \phi}\right| / H^{2}$ as a function of $\xi$ for the CDL instanton solution of Model 2. We can see that for most of the trajectory the condition $\left|V_{\phi \phi}\right|>H^{2}$ is satisfied. condition $\left|V_{\phi \phi}\right|>H^{2}$ is indeed satisfied for most of the trajectory.

Qualitatively, the features of Model 2 are very similar to Model 1, except that the dependence of the potential on hyperbolic trigonometric functions makes deviations from the fiducial $m^{2} \phi^{2}$ potential around the tunneling barrier sharper and more localized. Given the similarities, we refrain from reproducing the full set of plots given in the case of Model 1, focusing only on those we feel highlight the differences between the two models.

In order to highlight the sharpness of the barrier feature, in Fig. 10 we plot Model 2's equivalent of Fig. 5. Namely, we plot the potential, the corresponding fiducial $m^{2} \phi^{2}$ potential and the position of the field at the time of potential-curvature equality, the time that $p_{H_{0}}$ left the horizon and the time that $p_{100}$ left the horizon. We see that the potential relaxes to the fiducial one at around the time that $p_{H_{0}}$ left the horizon, which is much sooner than in the case of Model 1. As such, we expect that a much smaller range of scales will be subject to suppression.

In Fig. 11 we plot the scalar and tensor power spectra as a function of $p$, with the same set of curves included here as in Fig. 7. Regarding the scalar spectrum, in the case of Model 2 we find that using $c_{1}=3.5$ in Eq. (14) gives a better fit to the numerical results of Linde et al. for small p. Once again we find that at intermediate scales, i.e. $p \simeq 10$, the analytic fit differs from the numerical results by an amount on the order of $10 \%$. We also see that neglecting the $p$-dependent suppression factor in Eq. (14) and using only the naive horizon-crossing condition to determine $t_{\mathcal{R}, p}$ have very similar effects as in the case of Model 1 . The cutoff spectrum represented by the solid orange curve is constructed in exactly the same way as in the case of Model 1. As with Model 1, we find that the spectrum of Model 2 is much more suppressed on large scales compared to the cutoff model. A tensor contribution to CMB temperature fluctuations could therefore be accommodated in the case that the results of BICEP2 are confirmed. In comparison

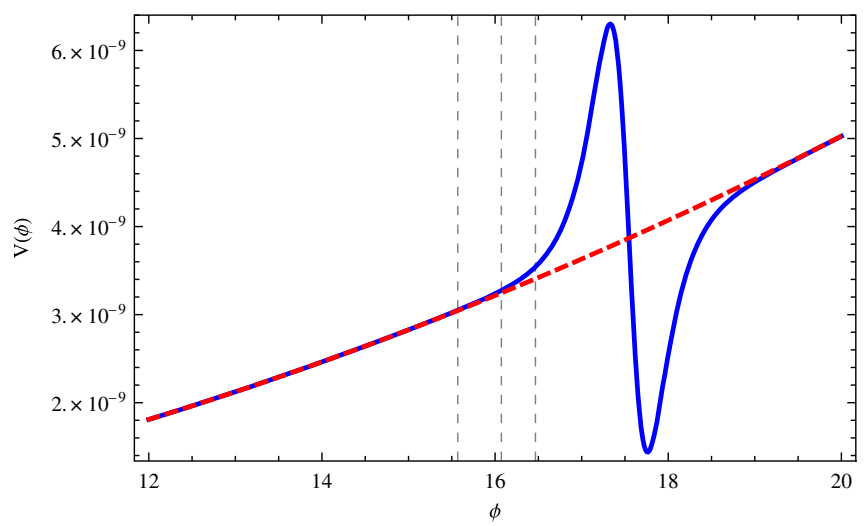

FIG. 10 (color online). Plot of the potential in Model 2 of Ref. [24] with the parameter choice shown in the main text. The three vertical lines are as in Fig. 5. 
SCALAR SUPPRESSION ON LARGE SCALES IN OPEN ...

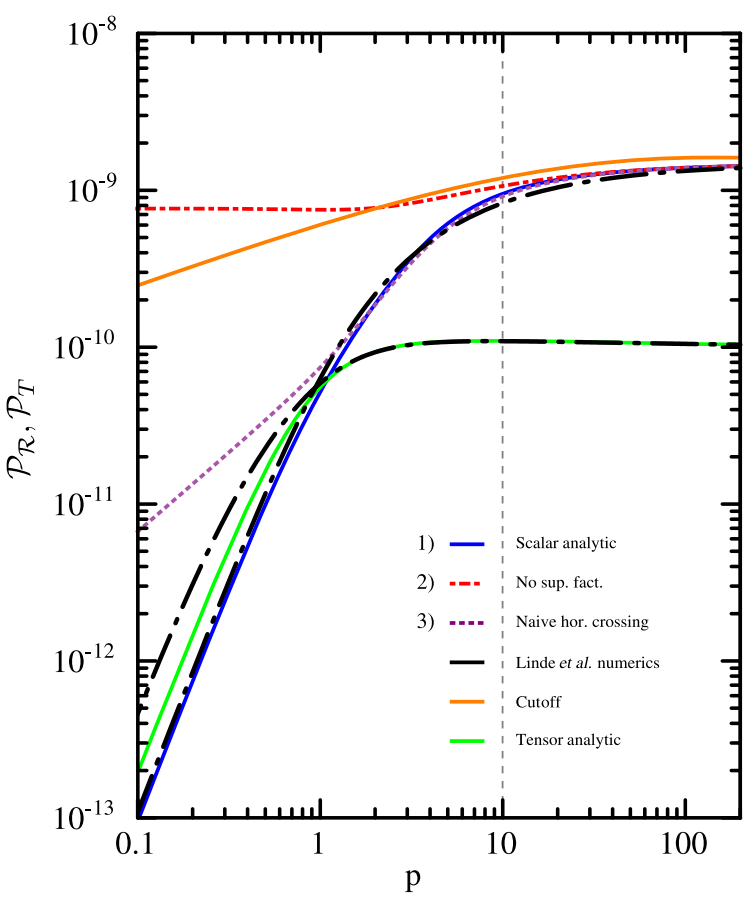

FIG. 11 (color online). The scalar and tensor power spectra as a function of the comoving scale $p$ for Model 2. The set of curves plotted is the same as in Fig. 7 for Model 1, except that solid black curves are now replaced with black dot-dashed curves.

with Model 1, Model 2 is in much better agreement with the cutoff spectrum on smaller scales. Regarding the tensor spectrum, we once again use $c_{2}=1$ in our fit, and again we can see that suppression of the tensor spectrum only becomes active at much lower $p$ than in the case of the scalar spectrum.

Finally, in Fig. 12 we plot the magnitude of the scalar power spectrum relative to its value at the scale of the transition between a blue- and a red-tilted spectrum. It is in this plot that the differences between Models 1 and 2 become most apparent. Comparing with Fig. 8 we see that the transition from a blue- to a red-tilted spectrum occurs a few $e$-foldings earlier in Model 2, approximately 60 $e$-foldings before the end of inflation. Given that potentialcurvature equality occurs approximately $66 e$-foldings before the end of inflation, this means that we only have around $6 e$-foldings of the "fast-roll" phase. Correspondingly, a suppression of the scalar power spectrum is observable over a much smaller range of scales, with the smallest affected scales being quite close to $p_{100}$ in the case that $\Omega_{\mathrm{K}}=0.01$. Nevertheless, the model is still able to give rise to a suppression on observationally relevant scales while also satisfying constraints on $\Omega_{\mathrm{K}}$. Unlike Model 1, however, we see that if $\Omega_{\mathrm{K}}$ is of nondetectable magnitude, i.e. $\Omega_{\mathrm{K}} \lesssim 10^{-4} \rightarrow p_{H_{0}} \gtrsim 10^{2}$, then this model likely gives no suppression on observable scales. Similarly, if we were to make the standard assumption that the current Hubble scale left the horizon
PHYSICAL REVIEW D 90, 083517 (2014)

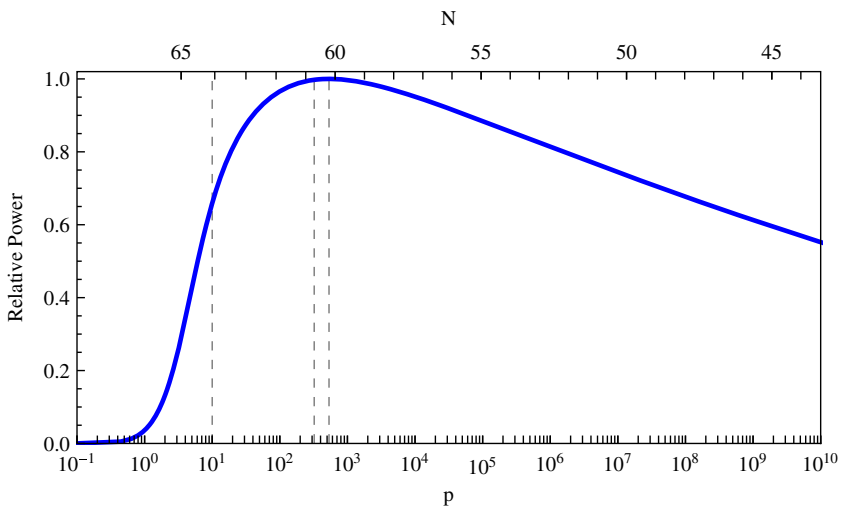

FIG. 12 (color online). The magnitude of the scalar power relative to the magnitude at the scale of transition between a blueand a red-tilted spectrum for Model 2. In this plot the vertical dashed lines, from left to right, correspond, respectively, to (1) the current horizon scale $p_{H_{0}}$ as determined assuming that $\Omega_{\mathrm{K}}=$ 0.01 , (2) the scale associated with $l=100, p_{100}$, and (3) the scale at which the spectrum transitions from being blue- to red-tilted, $p_{\text {red }}$. On the upper horizontal axis we also give the number of $e$-foldings before the end of inflation that each given scale $p$ leaves the horizon.

$\sim 60 e$-foldings before the end of inflation, then we see that there would be no suppression on observable scales.

\section{GENERAL CONSTRAINTS}

As discussed in the Introduction, our motivation for considering open inflation models is that they naturally give rise to a fast-roll phase between tunneling from the false vacuum and the onset of slow-roll inflation, which in turn leads to a suppression of the scalar power spectrum for modes leaving the horizon during this period. However, in order for this suppression to be observable, we require that the total number of $e$-foldings of inflation be relatively small. This in turn means that the curvature of space may not get diluted enough to satisfy current observational constraints on $\Omega_{\mathrm{K}}$. In this section we summarize how observational constraints on these two competing effects might be used to constrain models of open inflation.

As confirmed in the toy models of the preceding section, in general we expect that the dynamics can be considered in three stages. The first is the curvature-dominated stage, where $H=1 / a$, such that $a=t$ and $\dot{\phi} \simeq-V_{\phi} t / 4$. During this stage the Hubble friction term is large, meaning that $\dot{\phi}$ is very small. After the time $t_{*}$, defined by $V\left(\phi_{*}\right) / 3=$ $1 / a_{*}^{2}$, the potential energy comes to dominate over the curvature term. In the initial potential-dominated stage the slope of the potential may still be somewhat steeper than usually dictated by the requirement for slow roll. As such, in this stage we might expect $\dot{\phi}$ to be somewhat enhanced, leading to a suppression of the scalar power spectrum for modes leaving the horizon during this period. Finally, as the field evolves away from the barrier, the 
potential flattens and usual slow-roll inflation occurs. The above scenario is depicted schematically in Fig. 13.

In addition to the evolution of the Hubble parameter, Fig. 13 shows the evolution of the physical length scales associated with the curvature scale, $p_{\mathrm{K}}=1$, the current Hubble scale, $p_{H_{0}}$, and the scale associated with the onset of power suppression, $p_{\text {sup }}$. These scales can all be constrained using observations, which allows us to determine the relative position of the corresponding curves on the plot. For a given open inflation model, we would then require that the time at which the scale associated with the onset of suppression leaves the horizon coincides with the onset of slow-roll inflation.

To determine the separation between the curvature scale and the current Hubble scale, we note that the current observational constraint on $\Omega_{\mathrm{K}} \equiv 1 /\left(a_{0} H_{0}\right)^{2}$ is $\Omega_{\mathrm{K}} \lesssim 0.01$. We therefore find $\ln \left(p_{H_{0}} / p_{\mathrm{K}}\right) \gtrsim \ln (10) \simeq 2.3$, which corresponds to the vertical spacing between the blue and red lines in Fig. 13.

Next, in order to determine an estimate for the separation between the current Hubble scale and the scale at which the onset of scalar suppression is observed, we use the results of a recent analysis by Easther et al. [8]. In this work they considered a "broken" spectrum that is red-tilted up to some given scale $p_{\text {sup }}$ and then blue-tilted for larger scales, thus explaining the suppression on large scales. The transition is assumed to take place instantaneously at

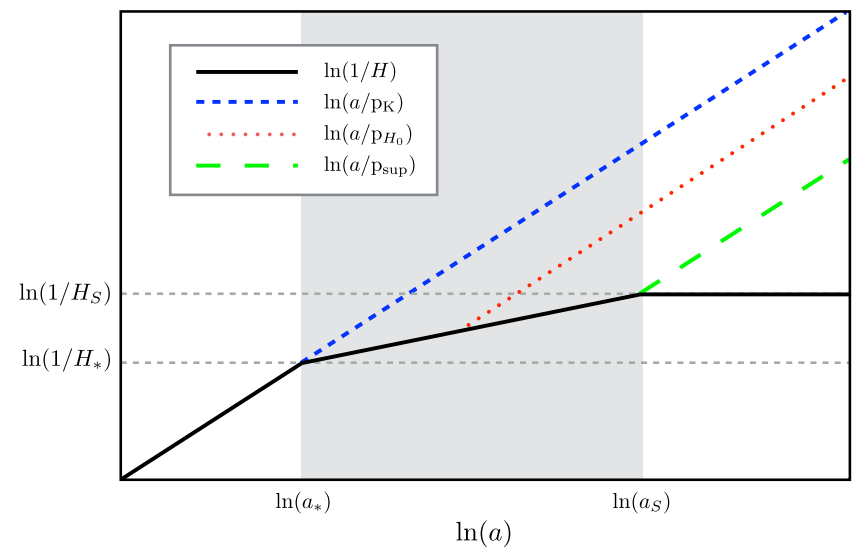

FIG. 13 (color online). Schematic diagram showing the evolution of $\ln (1 / H)$ (solid black curve) through the three stages of post-tunneling evolution referred to in the main text. Initially the curvature dominates, giving $1 / H \propto a$. In the second stage the potential now dominates but the slope of the potential is too steep to allow for slow-roll inflation, so the Hubble rate continues to evolve, but less rapidly. Once the potential flattens out, a period of slow-roll inflation commences, where the Hubble rate is approximately constant. Also plotted are the curvature scale (blue dashed curve), the current Hubble scale (red dotted curve) and the scale associated with the onset of suppression (green longdashed curve). The points at which these curves intersect the solid black curve corresponds to the horizon-crossing times. The shaded region corresponds to the fast-roll period. $p=p_{\text {sup }}$, and the best fit is found to be $p_{\text {sup }} / a_{0}=$ $4.6 \times 10^{-3} \mathrm{Mpc}^{-1}$, which can be compared to the value of $p_{H_{0}} / a_{0}=(h / 3) \times 10^{-3} \mathrm{Mpc}^{-1} \simeq(0.23) \times 10^{-3} \mathrm{Mpc}^{-1}$. We thus find $\ln \left(p_{\text {sup }} / p_{H_{0}}\right) \simeq 2.98$, which corresponds to the vertical separation between the red and green lines in Fig. 13.

Combining the above results, we find $\ln \left(p_{\text {sup }} / p_{\mathrm{K}}\right) \gtrsim 5.28$, which corresponds to the total vertical separation between the blue and green lines in Fig. 13. Of course, we stress that this is a very simple estimate, as defining and constraining the onset of suppression is nontrivial and model dependent.

By considering the evolution of the Hubble parameter during the transition from curvature domination to slowroll inflation, we are able to convert this constraint on the separation in scales to constraints on the potential. Let us use the subscripts " $*$ " and " $S$ " to denote quantities at the time of curvature-potential equality and the onset of slow-roll inflation, respectively. Then, using the definition of $\epsilon \equiv d \ln H / d N$, we have

$$
H_{S}=H_{*} \exp \left[\int_{*}^{S} \epsilon d N\right]
$$

By definition, we also have $a_{S}=a_{*} \exp [\Delta N]$, where $\Delta N$ is the number of $e$-foldings between curvature-potential equality and the onset of slow-roll inflation. As such, for scales associated with the onset of power suppression, i.e. scales leaving the horizon at the time of the transition between the fast- and slow-roll phases, we have

$$
p_{\text {sup }}=a_{S} H_{S}=a_{*} H_{*} \exp \left[\Delta N+\int_{*}^{S} \epsilon d N\right] \text {, }
$$

where we have made the simplifying assumption that the horizon-crossing condition $p=a H$ is valid, which should be true for these scales. We then note that prior to the time $t_{*}$ we have curvature domination, during which $a H=1=p_{\mathrm{K}}$. As such, we can rewrite the above expression as

$$
\Delta N+\int_{*}^{S} \epsilon d N=\ln \left(\frac{p_{\text {sup }}}{p_{\mathrm{K}}}\right)=\ln \left(\frac{p_{\text {sup }}}{p_{H_{0}}}\right)+\ln \left(\frac{1}{\sqrt{\Omega_{\mathrm{K}}}}\right) .
$$

Inserting the numbers discussed above, this gives

$$
\Delta N+\int_{*}^{S} \epsilon d N \gtrsim 5.3 .
$$

In the case that $\epsilon$ is constant during the fast-roll phase (as is the case in Fig. 13), this simplifies further to

$$
\Delta N(1-\epsilon)=\ln \left(\frac{H_{*}}{H_{S}}\right) \frac{1-\epsilon}{\epsilon} \gtrsim 5.3 .
$$

Another way in which we can try to constrain the form of the potential after the tunneling is by looking at how much 
the large-scale power spectrum is suppressed. Taking the naive relation $\mathcal{P}_{\mathcal{R}} \propto H^{2} / \epsilon$, and further neglecting the variation in $H$, the ratio of the power spectrum on large scales to that on small scales will tell us about the ratio of the slow-roll parameters, i.e.

$$
\frac{\mathcal{P}_{\mathcal{R}}\left(p_{1}\right)}{\mathcal{P}_{\mathcal{R}}\left(p_{2}\right)} \simeq \frac{\epsilon_{p_{2}}}{\epsilon_{p_{1}}} \leq 1
$$

where $p_{1} \leq p_{2}$ and $\epsilon_{p_{i}}$ indicates the value of $\epsilon$ when the scale $p_{i}$ left the horizon. In the schematic example depicted in Fig. 13, this approach is particularly simple as $\epsilon$ simply takes on two different but constant values in the fast- and slow-roll stages. As we have seen in Models 1 and 2, however, in more realistic models the slow-roll parameter is likely to vary with time during the fast-roll phase, so that the suppression becomes scale dependent. Furthermore, we have seen that the fast rolling of the field is not the only source of suppression for small $p$. The additional suppression from quantum tunneling effects is not captured in this simplified analysis.

\section{CONCLUSIONS}

The latest CMB measurement from WMAP and Planck hint at there being a deficit in the temperature power spectrum on large scales, and the possible detection of primordial gravitational waves by the BICEP 2 team only stands to compound this tension. In light of this, the fact that open inflation models give rise to a suppression of scalar power on large scales has led to them receiving renewed interest. Intuitively, the main source of suppression in these models is the steepening of the potential towards the barrier that separates the true and false vacuums. This steepening leads to a fast-roll phase in which $\dot{\phi}$ is enhanced, so that $\mathcal{P}_{\mathcal{R}} \propto H^{4} / \dot{\phi}^{2}$ becomes suppressed. The requirement that this fast-rolling should affect observable scales puts a limit on the duration of ensuing slow-roll inflation. If inflation does not last long enough, however, then one risks violating observable constraints on $\Omega_{\mathrm{K}}$.

In this paper we have revisited two of the toy models of single-field open inflation introduced in Ref. [24], with potentials given by Eqs. (20) and (25). We have seen that both models are indeed capable of giving suppression of the scalar power on the order of $10 \%$, and that they are also able to evade current constraints on $\Omega_{\mathrm{K}}$. The steepening of the potential towards the barrier in Model 1 was of powerlaw form, and this gradual steepening meant that the onset of suppression was also gradual, potentially affecting 3 orders of magnitude of observable scales. In Model 2, however, the steepening was of exponential form, leading to a much shorter fast-roll phase. Correspondingly, the range of affected observable scales was much narrower.

Finally, in both models we found that in addition to the source of suppression mentioned above, one also has additional suppression that results from the system's memory of the tunneling phase. (See also Ref. [24].) In the case that current bounds on $\Omega_{\mathrm{K}}$ are almost saturated, we saw that this additional suppression can be non-negligible on observable scales. As such, any quantitative analysis would need to take into account this additional suppression.

\section{ACKNOWLEDGMENTS}

We would like to thank R. Saito, K. Sugimura, T. Tanaka and D. Yeom for valuable discussions. This work was supported by the JSPS Grant-in-Aid for Scientific Research (A) No. 21244033.
[1] G. Hinshaw et al. (WMAP Collaboration), Astrophys. J. Suppl. Ser. 208, 19 (2013).

[2] P. Ade et al. (Planck Collaboration), arXiv:1303.5076 [Astron. Astrophys. (to be published)].

[3] P. Ade et al. (Planck Collaboration), arXiv:1303.5082.

[4] P. Ade et al. (Planck Collaboration), arXiv:1303.5075.

[5] P. Ade et al. (BICEP2 Collaboration), Phys. Rev. Lett. 112, 241101 (2014).

[6] R. Bousso, D. Harlow, and L. Senatore, arXiv:1404.2278.

[7] K. M. Smith, C. Dvorkin, L. Boyle, N. Turok, M. Halpern, G. Hinshaw, and B. Gold, Phys. Rev. Lett. 113, 031301 (2014).

[8] K. N. Abazajian, G. Aslanyan, R. Easther, and L. C. Price, J. Cosmol. Astropart. Phys. 08 (2014) 053.

[9] D. K. Hazra, A. Shafieloo, G. F. Smoot, and A. A. Starobinsky, J. Cosmol. Astropart. Phys. 06 (2014) 061.

[10] J.-O. Gong, J. Cosmol. Astropart. Phys. 07 (2014) 022.
[11] M. Gerbino, A. Marchini, L. Pagano, L. Salvati, E. Di Valentino, and A. Melchiorri, Phys. Rev. D 90, 047301 (2014).

[12] C. R. Contaldi, M. Peloso, L. Kofman, and A. D. Linde, J. Cosmol. Astropart. Phys. 07 (2003) 002.

[13] J. M. Cline, P. Crotty, and J. Lesgourgues, J. Cosmol. Astropart. Phys. 09 (2003) 010.

[14] B. A. Powell and W. H. Kinney, Phys. Rev. D 76, 063512 (2007).

[15] M. Kawasaki, T. Sekiguchi, T. Takahashi, and S. Yokoyama, J. Cosmol. Astropart. Phys. 08 (2014) 043.

[16] R. K. Jain, P. Chingangbam, J.-O. Gong, L. Sriramkumar, and T. Souradeep, J. Cosmol. Astropart. Phys. 01 (2009) 009.

[17] R. K. Jain, P. Chingangbam, L. Sriramkumar, and T. Souradeep, Phys. Rev. D 82, 023509 (2010). 
[18] F. G. Pedro and A. Westphal, J. High Energy Phys. 04 (2014) 034.

[19] D. K. Hazra, A. Shafieloo, G. F. Smoot, and A. A. Starobinsky, Phys. Rev. Lett. 113, 071301 (2014).

[20] R. Bousso, D. Harlow, and L. Senatore, arXiv:1309.4060.

[21] B. Freivogel, M. Kleban, M. Rodriguez Martinez, and L. Susskind, J. High Energy Phys. 03 (2006) 039.

[22] B. Freivogel, M. Kleban, M. R. Martinez, and L. Susskind, arXiv:1404.2274.

[23] A. D. Linde, Phys. Rev. D 59, 023503 (1998).

[24] A. D. Linde, M. Sasaki, and T. Tanaka, Phys. Rev. D 59, 123522 (1999).

[25] S. R. Coleman and F. De Luccia, Phys. Rev. D 21, 3305 (1980).

[26] L. G. Jensen and P. J. Steinhardt, Nucl. Phys. B237, 176 (1984).
[27] S. R. Coleman, V. Glaser, and A. Martin, Commun. Math. Phys. 58, 211 (1978).

[28] M. Sasaki, T. Tanaka, and K. Yamamoto, Phys. Rev. D 51, 2979 (1995).

[29] D. Yamauchi, A. Linde, A. Naruko, M. Sasaki, and T. Tanaka, Phys. Rev. D 84, 043513 (2011).

[30] K. Sugimura and E. Komatsu, J. Cosmol. Astropart. Phys. 11 (2013) 065.

[31] J. Garriga, X. Montes, M. Sasaki, and T. Tanaka, Nucl. Phys. B551, 317 (1999).

[32] K. Yamamoto, M. Sasaki, and T. Tanaka, Phys. Rev. D 54, 5031 (1996).

[33] J. Garriga, X. Montes, M. Sasaki, and T. Tanaka, Nucl. Phys. B513, 343 (1998).

[34] A. R. Liddle and S. M. Leach, Phys. Rev. D 68, 103503 (2003). 